\title{
Electrochemical and optical properties of two dimensional electrostatic assembly of Au nanocrystals
}

\author{
Joseph J. Kakkassery, Jean-Pierre Abid, Michel Carrara and David J. Fermín* \\ Laboratoire d'Electrochimie Physique et Analytique, Institut de Chimie Moléculaire et \\ Biologique, Ecole Polytechnique Fédérale de Lausanne, CH-1015 Lausanne, Switzerland. \\ E-mail: david.fermin@epfl.ch; Fax: +41 21 6933667; Tel: +41 216933162
}

Received 27th March 2003, Accepted 2nd May 2003

First published as an Advance Article on the web 11th August 2003

The spectroscopic and electrochemical properties of two-dimensional electrostatic assembly of Au nanocrystals are examined on poly-L-lysine (pLys) modified gold electrodes. The surface preparation for the nanoparticle deposition involved the selfassembly of a monolayer of 11-mercaptoundecanoic acid on the electrode surface, followed by the electrostatic deposition of pLys from aqueous solution. The polyelectrolyte layer acts as the electrostatic anchor for the Au particles. Electrostatically stabilised Au particles were prepared by homogeneous reduction in the presence of citrate, yielding monodispersed colloidal suspension with an average diameter of $18 \pm 2 \mathrm{~nm}$. After $4 \mathrm{~h}$ of deposition, the citrate-stabilised particles reach a maximum surface density of $(8.2 \pm 0.1) \times 10^{10}$ particles $\mathrm{cm}^{-2}$, with an average edge-to-edge distance of $25 \mathrm{~nm}$. The particle surface density was estimated from scanning electron micrographs. Kelvin probe measurements were employed for examining changes in surface dipole introduced by the $2 \mathrm{D}$ array of nanocrystals. From simple electrostatic arguments, the apparent static dipole moment per particle was estimated of the order of $2700 \mathrm{D}$. The strong interaction between the nanocrystals and the pLys layer is responsible for the surface charge displacement, leading to changes in the surface dipole of $0.35 \mathrm{eV}$. These electrostatic interactions also manifest itself by the red shift of the plasmon resonance of the assembly with respect to the aqueous colloidal suspension. Analysis of the spectral broadening was attempted within the framework of the so-called coherent-potential approximation. Finally, electrochemical studies in 1,2-dichloroethane show a large electronic overlap between the nanocrystals and the metal substrate. Results obtained from electrochemical impedance spectroscopy strongly suggest that the electrostatic assembly of nanocrystal behaves like a 2D array of randomly distributed spherical nanoelectrodes.

\section{Introduction}

The assembly of nanocrystals with a narrow size distribution on two and three-dimensional arrays is a key step in the development of nanoparticle based electronic components. ${ }^{1-4}$ Control over the nanocrystal size as well as interparticle and particle-substrate distance open up the possibility of designing junctions featuring mono-electron conductivity. ${ }^{3-5}$ Various methods have been employed for assembling colloidal particles at electrode surfaces based on the properties of the "anchoring" species. The most common approaches entail chemical bonding (e.g. S-Au), ${ }^{6-11}$ van der Waals 
forces involving the stabilisers at the particle surface, ${ }^{12-16}$ and electrostatic forces. ${ }^{17-21}$ Although tremendous efforts have been devoted to the characterisation of nanoparticle arrays obtained by different assembling methods, systematic studies of the size and nature of the nano-object, deposition time, particle-substrate distance and so forth are somewhat scarce. The present contribution delivers our initial efforts towards establishing detailed correlation between the organisation of the assembly and the optical and electrochemical properties.

The spectroscopic features of $\mathrm{Au}$ nanocrystals in the visible domain are determined by the interband transitions and the collective excitation of conduction band electrons (plasmon). The latter is affected not only by the dielectric properties of the surrounding media but also by the size of the nanocrystals and the density of electrons. ${ }^{22-24}$ The absorption features associated with the plasmon resonance in an ensemble of particles contain information on the particle distribution as well as on the effective dielectric properties of the film. In the case of assemblies on electrode surfaces, the effect of the potential on the plasmon intensity manifest itself by a homopolar electroreflectance signal as demonstrated by Baum et al. ${ }^{25}$ and Sagara et al. ${ }^{17}$ These studies demonstrated that the electron occupation in the nanoparticle assembly is determined by the position of the Fermi level of the solid. Furthermore, both works reported a red shift of the electroreflectance signal with respect to the plasmon resonance of the colloidal solution, suggesting significant changes in the effective dielectric properties of the surrounding medium and/or lateral particle interactions.

The electrochemical behaviour of $\mathrm{Au}$ assemblies has also been studied in connection to the dynamics of electron transfer ${ }^{21,26-28}$ and quantised double layer charging. ${ }^{28-32}$ Recent works by the groups of Murray and Chen have provided clear evidence of stepwise charging of the double layer at $\mathrm{Au}$ clusters of less than $2 \mathrm{~nm}$ diameter at room temperature, which is a phenomenon comparable to the so-called coulomb staircase. Cluster of these dimensions appear to exhibit measurable electron transfer rate constant to the underlying electrode surface of the order of $100 \mathrm{~s}^{-1} .{ }^{27}$ Chen and Pei have also shown that the electron transfer rate is affected by the length of the alkyl chain employed as stabiliser of the Au clusters. ${ }^{28}$

In the present paper, we shall study the deposition and properties of ensembles of Au nanoparticles on modified polycrystalline gold electrodes. The assembling process is driven by electrostatic forces between the citrate groups located at the particle surface and a poly-L-lysine layer deposited on the electrode surface. The density of particles as a function of the time of deposition was monitored ex situ by scanning electron microscopy and Kelvin probe measurements. The latter provide information on the changes of the surface dipole of the electrode in the presence of the $\mathrm{Au}$ nanocrystals. Average dipole moments for the nanocrystal of the order of $2700 \mathrm{D}$ can be extracted from the change in the work function at the maximum particle coverage. Absorption spectra of the particle assembly obtained in reflection mode exhibit a substantial broadening with respect to the spectrum of the colloidal suspension due to the interaction with the polyelectrolyte layer and the disordered nature of the ensemble. Finally, the electrochemical reactivity of the system can be rationalised as a $2 \mathrm{D}$ assembly of randomly distributed nanoelectrodes, featuring a strong electronic overlap with the underlying metal substrate.

\section{Experimental}

\subsection{Synthesis of Au nanoparticles}

All the reagents used were of analytical grade. Colloidal solution of citrate stabilised Au particles were synthesised employing the well-known Turkevich method. ${ }^{33}$ A $2.5 \times 10^{-6} \mathrm{~mol} \mathrm{dm}^{-3}$ solution of tetrachloroaurate salt $\left(\mathrm{HAuCl}_{4} \cdot 3 \mathrm{H}_{2} \mathrm{O}\right)\left(190 \mathrm{~cm}^{3}\right)$ is heated to $95^{\circ} \mathrm{C}$ under continuous stirring in a reflux and $10^{-2} \mathrm{dm}^{3}$ of $1 \%$ sodium citrate $\left(\mathrm{C}_{6} \mathrm{H}_{5} \mathrm{O}_{7} \mathrm{Na}_{3} \cdot 2 \mathrm{H}_{2} \mathrm{O}\right)$ solution is introduced. The solution is refluxed for $1 \mathrm{~h}$ to complete the formation of the colloids. A TEM image of the nanoparticles obtained by this method is shown in Fig. 1. The average diameter obtained over an ensemble of 100 particles was $18 \pm 2 \mathrm{~nm}$.

\subsection{Preparation of the polycrystalline gold electrodes}

The gold electrodes were fabricated by thermal evaporation on glass slides at a pressure of less than $5 \times 10^{-6}$ mbar (Edwards Auto 306). The glass slides were cleaned by sequential sonication in 


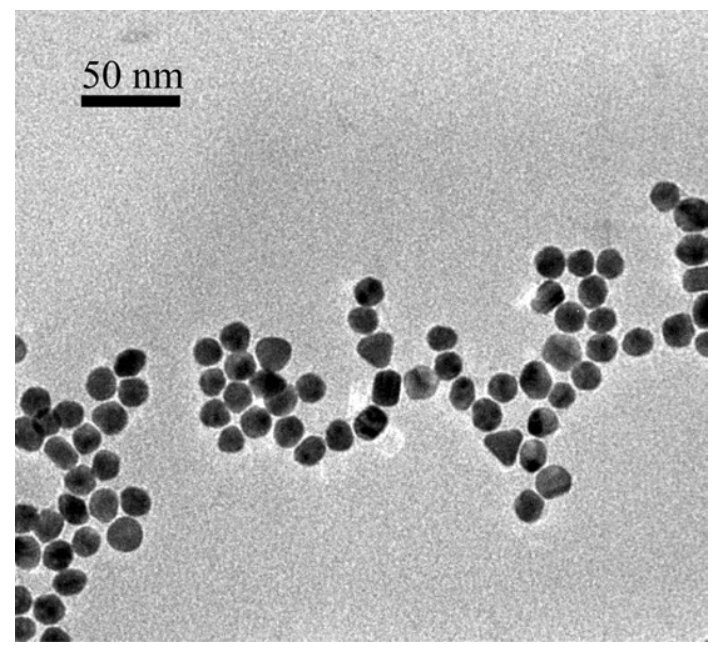

Fig. 1 TEM micrographs of the citrate stabilised Au nanocrystal featuring an average diameter of $18 \pm 2 \mathrm{~nm}$.

ethanol and acetone for 15 min each, followed by rinsing with copious amount of purified water in an ultrasound bath and dried under a stream of Ar. The evaporation procedure involved the growth of a $10 \AA$ thick layer of chromium (99.99\%, Balzers) at a rate of $1 \AA \mathrm{s}^{-1}$ to enhance the adhesion of the gold film. The gold film was initially grown at a rate of $1 \AA \mathrm{s}^{-1}$ up to $50 \AA$ and subsequently at $20 \AA \mathrm{s}^{-1}$ up to $1000 \AA$. The relatively high growth rate generates an amorphous gold film with a work function of $5.20 \pm 0.05 \mathrm{eV}$.

\subsection{Protocol for the assembly of nanoparticles}

The freshly prepared gold electrodes were dipped into an ethanolic solution of $10^{-3} \mathrm{~mol} \mathrm{dm}^{-3}$ 11-mercaptoundecanoic acid (MUA) for $12 \mathrm{~h}$. The MUA grafting time was established from measurements of the temporal evolution of the work function of the system. Kelvin probe measurements of gold slides as a function of the time of exposure to the MUA solution feature a monotonic increase of the work function, reaching a plateau after 10 hours. ${ }^{34}$ This behaviour indicates that the self-assembled monolayer (SAM) is formed inducing an average surface dipole pointing towards the metal surface, taking the dipole direction as from $\delta^{-}$to $\delta^{+}$. The excess of MUA molecules were removed from the electrode surface by rinsing with ethanol several times, washed with water and dried in an argon stream.

The SAM modified electrode was subsequently introduced into $1 \mathrm{~g} \mathrm{dm}^{-3}$ aqueous solution of poly-L-lysine (pLys) at $\mathrm{pH} 7$ for $30 \mathrm{~min}$. At this $\mathrm{pH}$, the carboxyl groups of the MUA layer are deprotonated, leading to an electrostatic induced deposition of pLys at the surface. ${ }^{35}$ The average thickness of this layer is of the order of $3 \mathrm{~nm} .{ }^{36}$ The electrode is subsequently removed and rinsed thoroughly with water to eliminate the excess of the polyelectrolyte. The cationic pLys layer plays the role of an electrostatic anchor to the negatively charged Au colloids. The subsequent steps involve the introduction of pLys modified electrode into the colloidal Au suspension for a given period of time. After the nanoparticle deposition, the electrode was copiously rinsed with water and dried under Ar prior to further characterisation.

\subsection{Electrochemical measurements}

Cyclic voltammetry and electrochemical impedance spectroscopy in the presence of ferrocene as redox couple were performed in 1,2-dichoroethane. The rationale behind the use of a hydrophobic solvent is to avoid the interpenetration of the electrolyte into the ultrathin layer assembly. The supporting electrolyte was bis(triphosphoranylidene)ammonium tetrakis(pentafluorophenyl)borate. The preparation of this electrolyte has been reported elsewhere. ${ }^{37}$ Two platinum wires were employed as pseudo-reference and secondary electrodes. The geometrical surface 
areas of the working and secondary electrodes were 1 and $3 \mathrm{~cm}^{2}$ respectively. All the potentials are referenced to as the formal redox potential of ferrocene, which corresponds to $(0.64 \pm 0.02) \mathrm{V}$ with respect to the standard hydrogen electrode. ${ }^{38}$

\subsection{Instrumentation}

Measurements of the work function were performed with a Kelvin probe (Delta Phi) in a glove box under Ar atmosphere and room temperature. A $10 \mathrm{~mm}$ diameter gold plate was used as reference. The average distance between the reference plate of the probe and the sample was kept at $c a .1 \mathrm{~mm}$ during the measurements. SEM images were obtained from a JEOL JSM 6300F microscope at 3 $\mathrm{kV}$ accelerating voltage. Absorption spectra were measured under reflection mode using an Arc-Xe lamp and a spectrograph Jobin-Yvon Spex TRIAX 190 Series fitted with a CCD detector (CCD3500). The electrochemical measurements were carried out with an Autolab PGSTAT12 system equipped with a frequency response analyser module. Electrochemical impedance spectra were collected in the frequency range between $1 \mathrm{~Hz}$ and $10 \mathrm{kHz}$, with an ac-potential amplitude of 10 $\mathrm{mV}$ rms.

\section{Results and discussions}

\subsection{Electrostatic assembly at pLys modified gold electrodes}

The work function $(\Phi)$ of the nanoparticle-modified electrode was monitored ex situ as a function of the deposition time by Kelvin probe measurements. By definition, $\Phi$ is determined by the chemical potential of electrons $\left(\mu_{\mathrm{e}}\right)$ and the surface potential $(\chi)$,

$$
-\Phi=\mu_{\mathrm{e}}-e \chi
$$

where $e$ is the elementary charge. The assembly of negatively charged particles leads to an increase of the surface potential as depicted in Fig. 2. Each point in the curve corresponds to a fresh sample in which the electrode was immersed for a fixed period of time in the colloidal suspension. Under

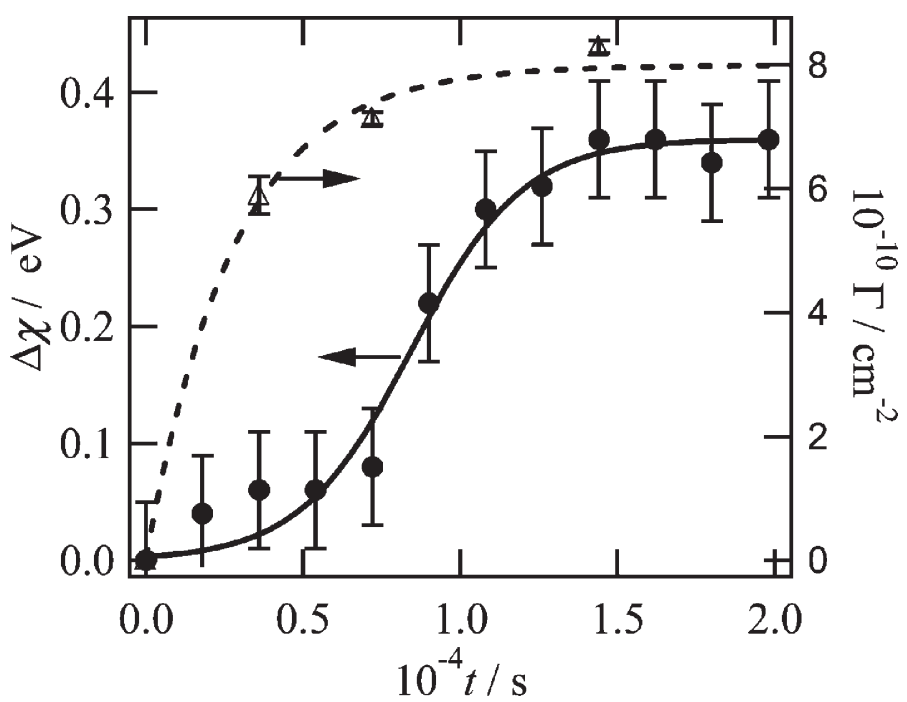

Fig. 2 Time dependence of the variation in the surface potential and particle density during the electrostatic assembly of the Au nanocrystals on pLys modified electrodes. The change in surface potential was estimated from Kelvin probe measurements (see text), while the surface density of particles was obtained from SEM micrographs (see Fig. 3). A maximum surface density of $(8.2 \pm 0.1) \times 10^{10}$ particles $\mathrm{cm}^{-2}$ was obtained after $4 \mathrm{~h}$ of deposition. 

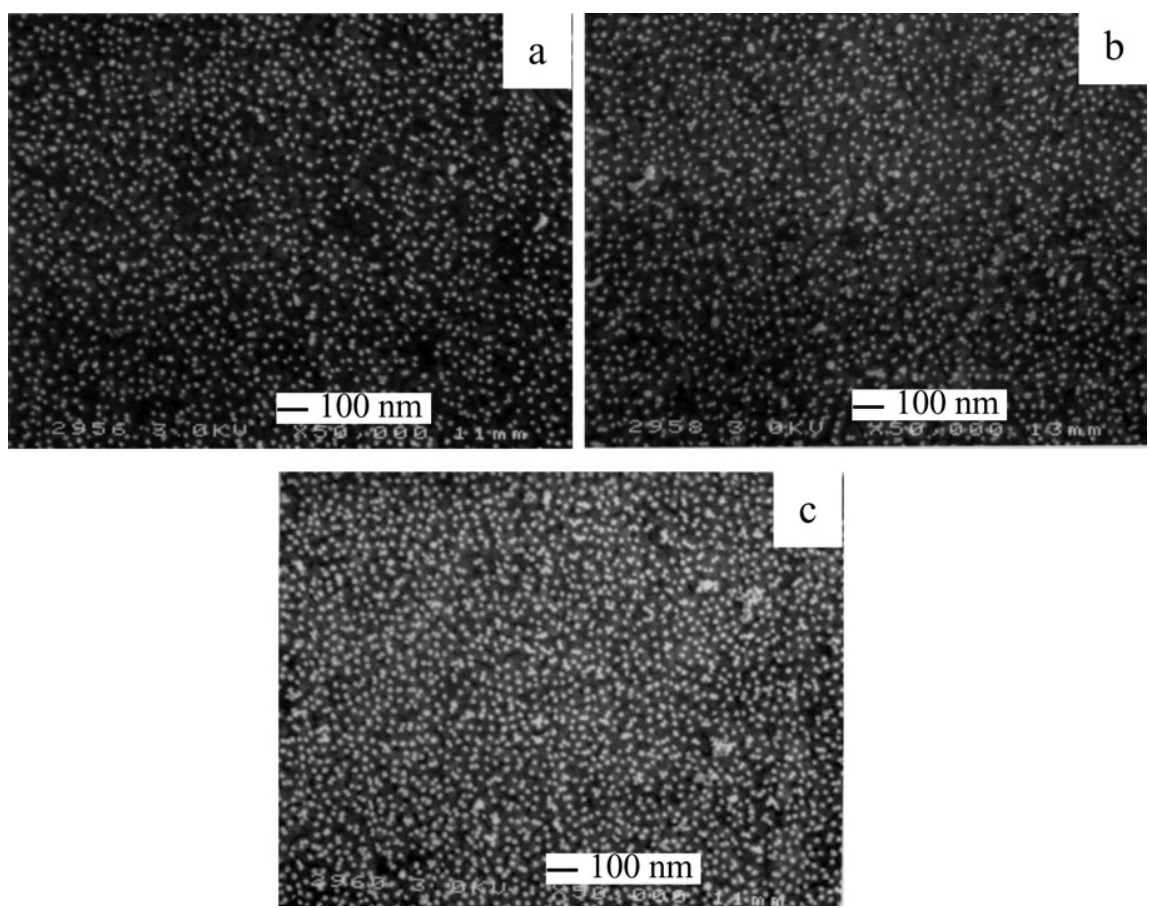

Fig. 3 SEM micrographs of the nanoparticle assembly after 1 (a), 2 (b) and $4 \mathrm{~h}$ (c) of deposition. The images show an increase in the surface density of particles. No significant changes were observed after $4 \mathrm{~h}$.

purely electrostatic grounds, the change of the surface potential $(\Delta \chi)$ can be expressed in terms of the average dipole moment $(\mu)$ and the particle surface density $(\Gamma)$

$$
\Phi_{\text {part }}-\Phi_{\text {film }}=\Delta \chi=\frac{e \Gamma \mu}{\varepsilon_{\mathrm{m}} \varepsilon_{0}}
$$

where $\varepsilon_{\mathrm{m}}$ and $\varepsilon_{0}$ correspond to the relative and vacuum permittivity respectively. From the results in Fig. 2, it can be observed that the particle density reaches a steady state value after approximately four hours of deposition. No significant changes in the work function were observed for deposition time of more than $24 \mathrm{~h}$.

The surface density of nanoparticles at the electrode surface was directly estimated from SEM pictures as illustrated in Fig. 3. The micrographs reveal that the particle density substantially increases after $60 \mathrm{~min}$ of deposition, reaching a maximum value after 4 hours. The size distribution extracted from the micrograph after $4 \mathrm{~h}$ deposition is depicted in Fig. 4a. The average size obtained from sampling 1980 particles resulted in $19.7 \pm 2.9 \mathrm{~nm}$ diameter, with less than $5 \%$ of large aggregates. The maximum particle density obtained from the SEM images was $(8.2 \pm 0.1) 10^{10}$ $\mathrm{cm}^{-2}$. As shown in Fig. $4 \mathrm{~b}$, the edge-to-edge distance between the particles shows a narrow distribution with a maximum close to $25 \mathrm{~nm}$. The interparticle distance reflects the electrostatic repulsion between the nanocrystals, which generates exclusion zones of dimensions comparable to the diameter of the particles.

As illustrated in Fig. 2, the coverage as a function of the deposition time does not follow the $t^{1 / 2}$ dependence expected for a diffusion controlled adsorption process. ${ }^{39}$ Assuming a Langmuir type of adsorption, the time dependent coverage $(\theta)$ can be simply expressed in terms of,

$$
\theta(t)=\frac{k_{\mathrm{a}}}{k_{\mathrm{a}}+k_{\mathrm{d}}}\left[1-\exp \left(-\left(k_{\mathrm{a}}+k_{\mathrm{d}}\right) / t\right)\right]
$$

where $k_{\mathrm{a}}$ and $k_{\mathrm{d}}$ are the phenomenological adsorption and desorption rate constants. The former is linearly dependent on the concentration of the particles in the suspension. The dashed line in Fig. 2 

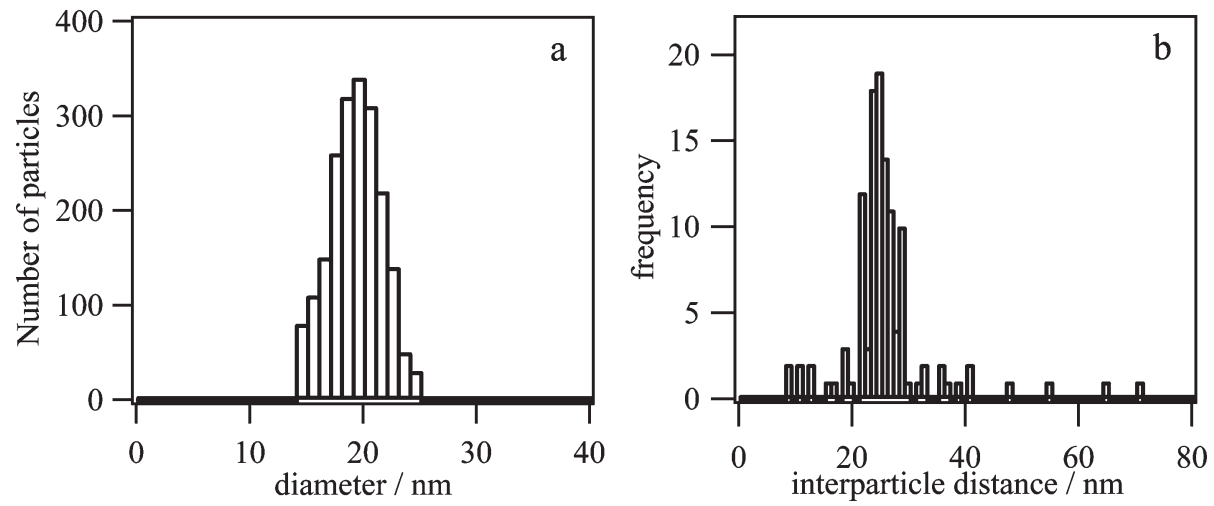

Fig. 4 Distribution of particle size (a) and edge-to-edge interparticle distance (b) obtained from the SEM image of the surface after 4 hours of deposition. It is observed lateral repulsion between the particles in the assembly induces a very low degree of aggregation and the appearance of an "exclusion zone" around the particles.

was obtained from eqn. (3) taking $k_{\mathrm{a}} / k_{\mathrm{d}}$ as 0.31 . The time dependences of $\Gamma$ and $\Delta \chi$ appear to indicate that the average dipole moment increases as the surface density increases. Taking a relative permittivity of the medium of 2.3, it can be extracted from eqn. (2) that $\mu$ increases from $c a$. 650 to $2700 \mathrm{D}$ as the surface density increases from $c a .4$ to $8 \times 10^{10} \mathrm{~cm}^{-2}$. The charge and dimension of the nanocrystals are the determining factors behind these large dipole moments. Evaluation of the colloidal mobility by capillary electrophoresis established an average charge per particle of $-1.0 \times 10^{-17} \mathrm{C}^{40}$ A dipole moment of the order of $2700 \mathrm{D}$ requires a displacement of $5 \%$ of the particle charge within the nanocrystalline domain. Furthermore, our most recent results also show that the increase in the surface dipole is substantially smaller for particles with an average diameter of $1 \mathrm{~nm} .{ }^{41}$ The value of the induced dipole moment in the particles depends on the value considered as the effective dielectric constant. The spectroscopic analysis discussed in the next section provides a rationale for taking $\varepsilon_{\mathrm{m}}=2.3$. The apparent increase of the average $\mu$ with increasing surface density could be interpreted in terms of a collective effect of the particle ensemble, i.e. that the effective dipole moment of the assembly is larger than the sum of the contribution of each particles at the surface. We are currently extending this analysis to nanocrystals of different size and nature in order to gain further information on the relationship between the apparent $\mu$ and the surface particle density.

\subsection{Plasmon band in the particle assembly}

The absorption spectra of the particles in the aqueous suspension as well as in the electrostatic assembly are contrasted in Fig. 5. The spectra of the assembly for different deposition time were measured in reflection mode at an angle of $34^{\circ}$ with respect to the normal, taking the reflection of the pLys modified gold electrode as reference. The aqueous suspension of the colloidal particle shows a well-defined plasmon resonance centred at $520 \mathrm{~nm}$, while the assembly features a significantly broader band with a maximum around $560 \mathrm{~nm}$. The light capture cross-section of the film increases as the time of deposition increases from 1 to $4 \mathrm{~h}$. Considering the polarisable nature of the metallic nanocrystals, the shift of the plasmon band can be interpreted in terms of changes in the dielectric properties of the medium and lateral interactions through dipolar fields. In this section, we shall attempt a semiquantitative analysis of the main spectroscopic features, i.e. the position of the plasmon resonance and the band broadening.

Various models have been developed in the literature for calculating the average polarisability $\alpha(\omega)$ of crystalline domains distributed in a dielectric medium $\varepsilon_{\mathrm{m}}$. One of the simplest approximation is given by the Maxwell-Garnet theory, which describes the effective dielectric function of a film featuring a random distribution of metallic spheres. ${ }^{42-44}$ In this model, the local field acting on a particle is given by the Lorenz relation. As disordering effects and local field fluctuations are neglected, this model cannot account for the broadening observed in the absorption spectrum of 


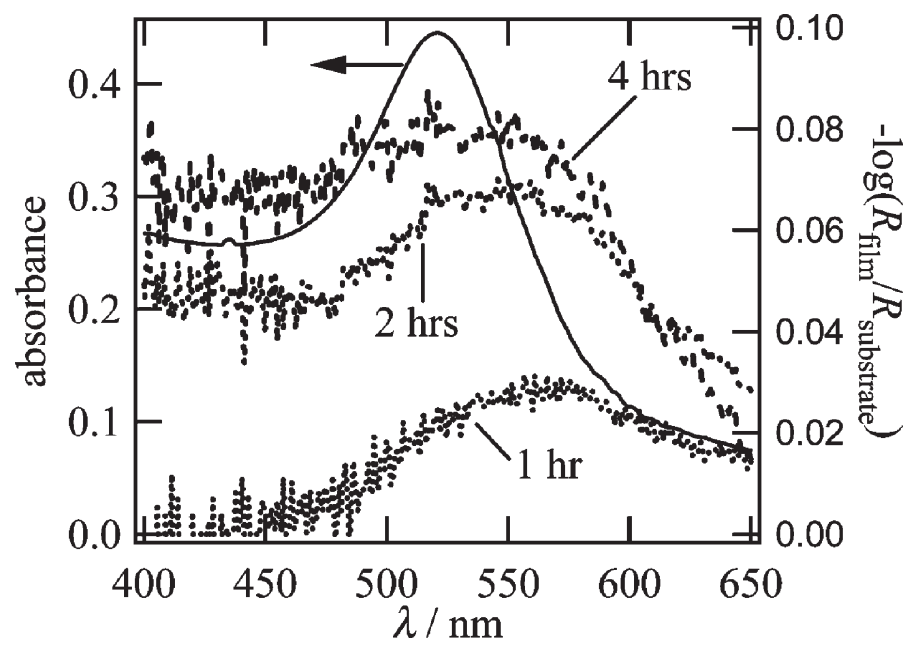

Fig. 5 Absorption spectra of the colloidal suspension (solid line) and the normalised reflectance of the electrostatic assembly after 1, 2 and $4 \mathrm{~h}$ of deposition (dashed lines).

the assembly. On the other hand, a more "realistic" approach is given by the so-called coherentpotential approximation (CPA). ${ }^{45-47}$ Considering that the polarisability of a particle located at the origin of the lattice corresponds to $\alpha_{0}(\omega)$, and the induced dipole moment due to an external field is $\vec{p}_{0}$, the CPA estimates the average dipole moment $\vec{p}=c \vec{p}_{0}$ on each of the nanocrystal sites of the lattice. The parameter $c$ is the fraction of sites occupied by the particles in the two dimensional array. The model establishes,

$$
\alpha(\omega)=c \alpha_{0}(\omega) /\left(1+\left(\alpha_{0}(\omega)-\alpha(\omega)\right) \sum_{\vec{q}} \frac{U(\vec{q})}{1+\alpha(\omega) U(\vec{q})}\right)
$$

where $\vec{q}$ is the position vector associated with the nanocrystalline sites in the assembly, and

$$
U(\vec{q})=\sum_{i \neq 0} \mathrm{e}^{\mathrm{i} q R_{\mathrm{i}}}\left(a^{2}-3 R_{i} R_{j}\right) a^{-5}
$$

corresponds to the Fourier transform of the dipole tensor. The summation in eqn. (5) determines the contribution of each of the particles in the array on the polarisability of the particle in the origin of the lattice, where $R_{i}$ corresponds to a position in the lattice. In order to simplify the resolution of eqn. (5), we shall assume a fcc structure of the array, which allows defining the parameter $c$ in terms of the volume density of particles $n$ as:

$$
n=4 c / l^{3}
$$

where $l$ is the lattice constant. In addition, we can also define the volume fraction occupied by the particles $f$ as,

$$
f=\frac{4 \pi n a^{3}}{3}
$$

This approximation implicitly redefined the system as a three dimensional assembly, where the effective thickness is determined by the diameter of the particles.

The polarisability of a sphere is associated with its diameter by the Laplace equation,

$$
\alpha(\omega)=\frac{\varepsilon_{\mathrm{k}}-\varepsilon_{\mathrm{m}}}{\varepsilon_{\mathrm{k}}+2 \varepsilon_{\mathrm{m}}} a^{3}
$$


where $\varepsilon_{\mathrm{k}}$ is the complex dielectric function that can be derived from the Drude model, ${ }^{24,48}$

$$
\begin{gathered}
\varepsilon_{\mathrm{k}}^{\prime}(\omega)=\varepsilon_{\mathrm{k}}^{\infty}-\frac{\omega_{\mathrm{p}}^{2}}{\left(\omega^{2}+\omega_{\mathrm{d}}^{2}\right)} \\
\varepsilon_{\mathrm{k}}^{\prime \prime}(\omega)=\frac{\omega_{\mathrm{d}} \omega_{\mathrm{p}}^{2}}{\omega\left(\omega^{2}+\omega_{\mathrm{d}}^{2}\right)}
\end{gathered}
$$

taking $\varepsilon_{\mathrm{k}}^{\infty}$ as the high frequency limit of the dielectric constant due to interband and core transitions, and $\omega_{\mathrm{p}}$ as the bulk plasma frequency which is determined by the concentration of free electrons in the metal $(N)$ and the effective mass of the electron $\left(m_{e}{ }^{*}\right)$,

$$
\omega_{\mathrm{p}}^{2}=N e^{2} / m_{e}{ }^{*} \varepsilon_{o}
$$

The relaxation damping frequency $\omega_{\mathrm{d}}$ is related to the mean free path of the conduction electrons $\left(L_{\mathrm{e}}\right)$ and the electron velocity at the Fermi energy $\left(v_{\mathrm{f}}\right)$,

$$
\omega_{\mathrm{d}}=v_{\mathrm{f}} / L_{\mathrm{e}}
$$

The extinction efficiency $\left(Q_{\text {ext }}\right)$ of the particles can be expressed in terms of the real and imaginary component of the dielectric function,

$$
Q_{\text {ext }}=\frac{24 \pi^{2} a^{3} \varepsilon_{\mathrm{m}}^{3 / 2}}{\lambda} \frac{\varepsilon_{\mathrm{k}}^{\prime \prime}}{\left(\varepsilon_{\mathrm{k}}^{\prime}+2 \varepsilon_{\mathrm{m}}\right)+\varepsilon_{\mathrm{k}}^{\prime \prime 2}}
$$

Analysis of the spectrum of the colloidal suspension (Fig. 5) based upon eqns. (9), (10) and (13) provided values for the parameters $\omega_{\mathrm{p}}, \varepsilon_{\mathrm{k}}^{\infty}$ and $\omega_{\mathrm{d}}$ of $9.2,8.2$ and $0.3 \mathrm{eV}$ respectively. The wavelength dependence of the arbitrarily normalised $Q_{\text {ext }}$ employing this set of parameters is displayed in Fig. 6. It is observed that the Drude model closely reproduces the essential features of the spectrum of the colloidal suspension. Knowledge of these parameters allows estimating the extinction cross section of the ensemble within the framework of the CPA model. The wavelength dependence of the arbitrarily normalised cross section of the assembly is also displayed in Fig. 6 as a function of $f$. As mentioned previously, this parameter is correlated to the particle distribution function contained in the summation in eqn. (5). It is observed that as $f$ increases, the broadening of the spectral responses drastically increases. The maximum of the spectral response is effectively

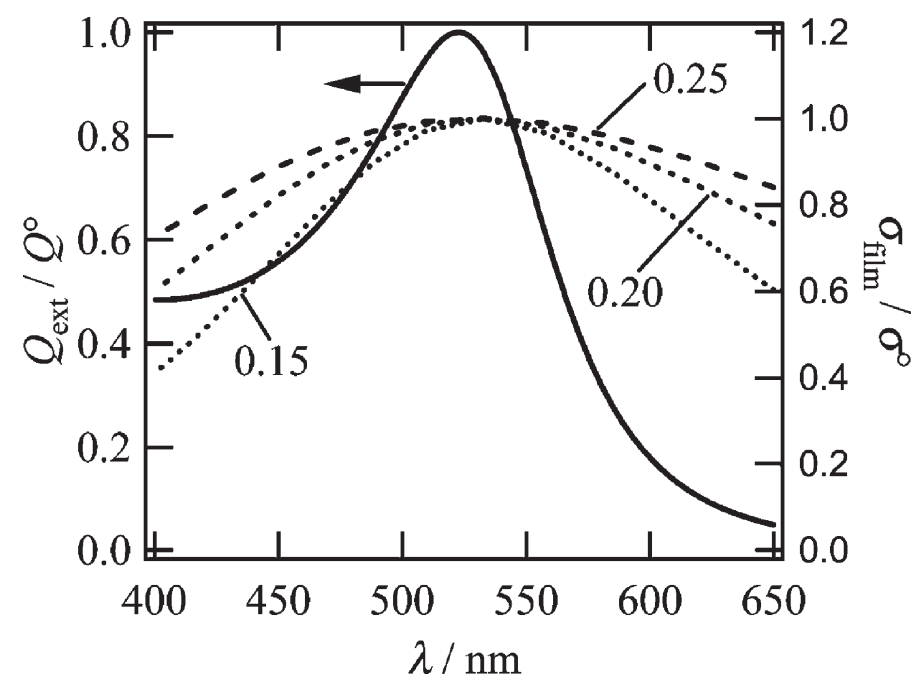

Fig. 6 Arbitrarily normalised extinction efficiency of the colloidal suspension (solid line) and extinction crosssection of the ensemble as estimated from the CPA model (dashed lines) for various particle volume fractions $f$. The values for $\omega_{\mathrm{p}}, \varepsilon_{\mathrm{k}}^{\infty}$ and $\omega_{\mathrm{d}}$ were taken as $9.2,8.2$ and $0.3 \mathrm{eV}$ respectively. The average dielectric constant of the film was taken as 2.3. 
independent of the parameter $f$ and is given by the resonance condition, i.e. the wavelength for which $\varepsilon_{\mathrm{k}}^{\prime}=-2 \varepsilon_{\mathrm{m}}$. The simulations show that the maximum of the spectra somewhat approaches the experimental values for $\varepsilon_{\mathrm{m}}=2.3$, which is close to the value reported for pLys. ${ }^{49}$ This result suggests that the polyelectrolyte layer exert a strong effect in the polarisability of the particles even if these are not embedded but "sitting" at the surface of the layer.

In relation to the spectral broadening, our preliminary analysis shows a substantial overestimation taking the average particle distance obtained from the SEM micrographs. From the micrographs in Fig. 3, it can be estimated that the values of $f$ are between 0.20 and 0.25 . However, the simulations in Fig. 6 predict spectral broadening similar to those observed experimentally for films with lower volume fraction. These differences do reflect the high complexity involved in these studies as well as limitations in the approach. To establish a more quantitative analysis of the spectral properties of the assembly, we would require a more accurate description of the particle distribution, extending beyond simply the average distance. We are currently performing more comprehensive spectroscopic studies in order to fully characterise the environment surrounding the particles in the assembly.

\subsection{Electrochemical properties of the nanocrystal assembly}

Cyclic voltammograms in DCE in the presence of ferrocene for the various stages in the preparation of the nanoparticle assembly are contrasted in Fig. 7. As expected for this redox couple, the cyclic voltammogram for the bare gold substrate indicates that the rate of oxidation is controlled by the diffusion of species to the electrode surface. Upon modification of the substrate by the SAM and pLys layer, the cyclic voltammograms exhibit a substantial increase in the peakto-peak separation with respect to the bare metal substrate. This behaviour indicates that the kinetic of oxidation is determined by the rate of electron transfer across the film at the formal transfer potential. The effect of SAM on the rate of electron transfer has been extensively studied in the literature, although the large majority of the works have been concentrated in aqueous electrolyte. Upon deposition of the Au nanocrystals for a period of $1 \mathrm{~h}$, the voltammetric features observed for the bare electrode are effectively recovered. Furthermore, no significant effects on the peak current are observed upon increasing time of deposition. These results indicate that the electron coupling between the particles in the ensemble and the electrode substrate is rather strong and that the current is effectively controlled by the diffusion of ferrocene to the nanoparticle assembly. A similar voltammetric behaviour has also been reported by Sagara et al. for Au nanoarrays assembled on aminoalkanethiol in aqueous electrolyte. ${ }^{17}$ Uosaki et al. have also
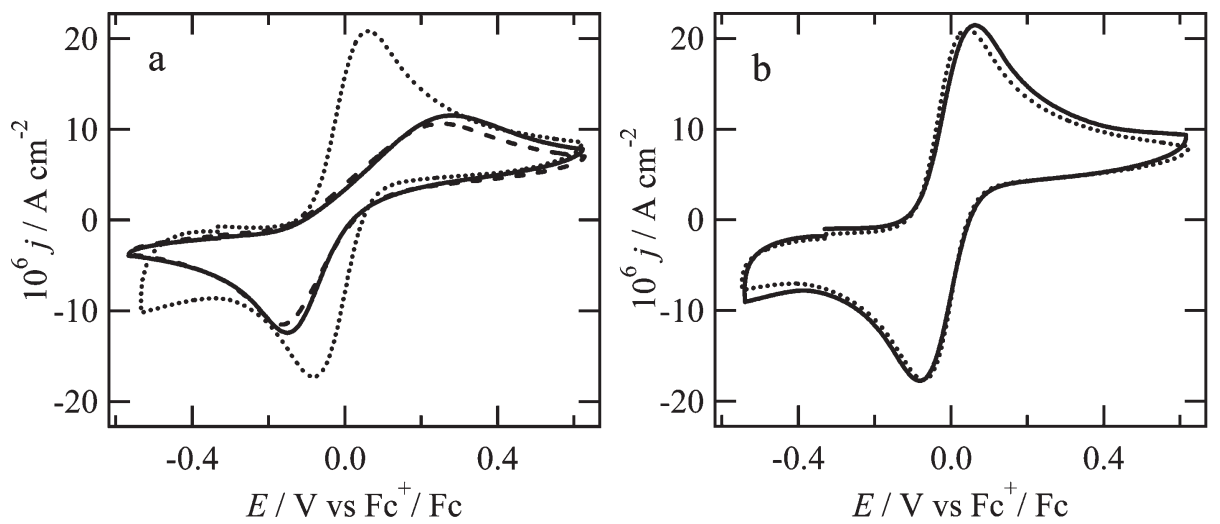

Fig. 7 Cyclic voltammograms of the bare gold substrate (dotted), MUA (full) and pLys (dashed) modified electrode at $50 \mathrm{mV} \mathrm{s}^{-1}$ in the presence of $10^{-4} \mathrm{~mol} \mathrm{dm}^{-3}$ ferrocene in 1,2-dichloroethane (a). The formation of the self-assembled monolayer and the subsequent deposition of the polycation significantly affect the dynamics of electron transfer. The cyclic voltammograms after 1 (solid) and $4 \mathrm{~h}$ (dotted line) of particle deposition show the same features as the bare Au substrate (b). 


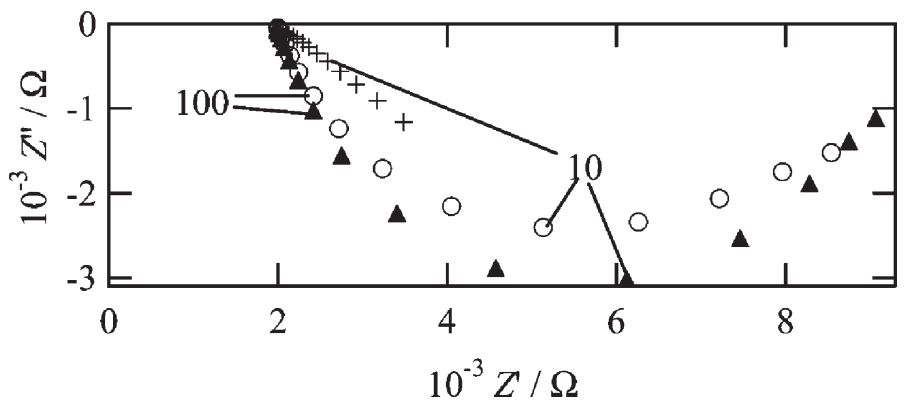

Fig. 8 Complex representation of the electrochemical impedance responses at the formal reduction potential of ferrocene for the MUA (circles), pLys (triangles) and Au nanocrystals (crosses) modified electrodes. The deposition time of the nanocrystals was $1 \mathrm{~h}$. The MUA and pLys modified electrodes exhibit a kinetic controlled electron transfer, while the oxidation on the nanoparticle assembly is limited by the ferrocene diffusion.

demonstrated that fast electron transfer can take place from ferrocene groups covalently bound to the gold nanocrystals. ${ }^{21}$

The trends observed in the voltammograms can be rationalised in further detail by the acimpedance spectra obtained at the formal redox potential of ferrocene. As shown in Fig. 8, the impedance responses for the nanoparticle-modified electrodes show a Warburg-type behaviour over the frequency range from $1 \mathrm{~Hz}$ to $10 \mathrm{kHz}$. By contrast, the impedance of the modified electrodes in the absence of the nanoparticles describes essentially Randles behaviour over most of the frequency range with charge transfer resistances of $(5.6 \pm 0.1) \times 10^{3}$ and $(6.2 \pm 0.1) \times 10^{3} \Omega$ for the SAM and pLys terminated surfaces respectively. The high values of the charge transfer resistance indicate the blocking nature of the SAM and pLys layer, which prevent the approach of the redox couple to the electrode surface. Although the organic solvent may affect the stacking of the alkyl chains in the SAM, it appears that the carboxyl termination inhibits the penetration of the redox species through the monolayer. The rather hydrophilic character of pLys increases the distance separating ferrocene to the metal substrate, further increasing the charge transfer resistance. However, this partial blocking effect is removed upon decorating the surface with $\mathrm{Au}$ nanocrystals. The fact that the impedance is determined by the diffusion of ferrocene to the modified electrode provides a clear indication of the strong electronic coupling between the substrate and the nanoparticles.

The impedance responses for the nanoparticle assembly at the formal transfer potential of the ferrocene oxidation remains very little affected by the time of deposition. The electron transfer is effectively "channelled" through the nanocrystals at the surface to the metal substrate, and this situation can be depicted as an array of "nanoelectrodes" distributed on an electrochemically inactive surface. In this case, the transient current responses should reflect a spherical mass diffusion profile around the nanocrystals at short times, developing to an overall planar geometry after the spherical diffusion profiles of neighbouring particles overlap

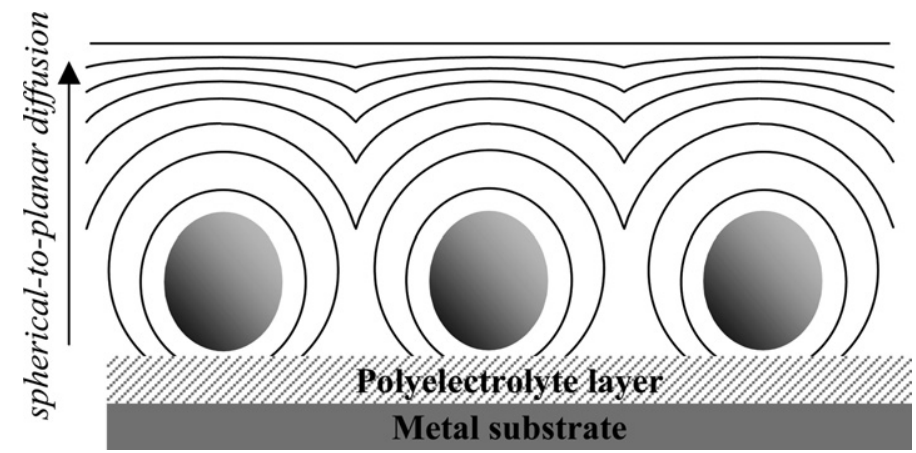

Fig. 9 Schematic representation of the overlap of the diffusion profiles generated around the particles in the $2 \mathrm{D}$ array. The transition from spherical to planar diffusion takes place at times shorter than $1 \mu \mathrm{s}$ (see text). 


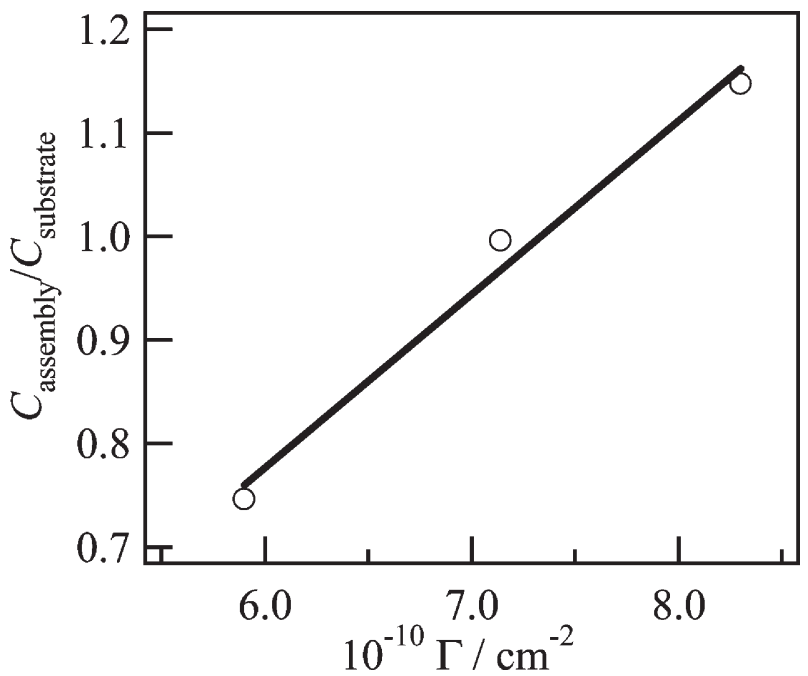

Fig. 10 Roughness factor as a function of the particle density at the modified electrode surface. The slope effectively corresponds to the average surface of a single particle. This result indicates that the exposed surface area of the particles in the assembly determines the double layer capacitance, i.e. the system approaches the behaviour of a 2D array of spherical nanoelectrodes.

in space. This phenomenon is schematically illustrated in Fig. 9. From the model developed by Scharifker ${ }^{50}$ for nanodisk arrays, the transition of spherical to planar diffusion takes place a time $\tau_{\mathrm{s}-\mathrm{p}}$

$$
\tau_{\mathrm{s}-\mathrm{p}}=\frac{\left(1-\pi \Gamma a^{2}\right)^{2}}{\pi D(\pi \Gamma a)^{2}}
$$

where $D$ corresponds to the diffusion coefficient. Evaluating eqn. (14) for $\Gamma$ of the order of $5 \times 10^{10} \mathrm{~cm}^{-2}$ and $D=10^{-5} \mathrm{~cm}^{2} \mathrm{~s}^{-1}$, it is obtained that the transition time is less than one microsecond. Consequently, the impedance measurements have to be extended into the $\mathrm{MHz}$ region in order to probe the spherical diffusion around the nanocrystals. Unfortunately, this frequency range is inaccessible under our current experimental conditions. The issue of addressing the electrochemical reactivity of single particles in $2 \mathrm{D}$ assemblies remains a highly controversial issue. ${ }^{51,52}$

Finally, we have examined the impedance responses at potentials more negative than the formal oxidation potential of ferrocene in order to evaluate the differential capacitance $\left(C_{\mathrm{diff}}\right)$ as a function of the number density of particles. Assuming that the effective surface area of the modified electrode is determined by the exposed area of the gold nanocrystals, it follows that:

$$
C_{\text {diff }} / C_{\text {diff }}^{\circ}=4 \pi a^{2} \Gamma
$$

where $C_{\text {diff }}{ }^{\circ}$ corresponds to the characteristic differential capacitance per unit area. Relation (15) corresponds to the roughness factor of the modified surface with respect to the metal substrate. The measured differential capacitance normalised by the capacitance of the bare Au substrate as a function of the particle density is illustrated in Fig. 10. The plot provides a linear relationship with a slope of $(1.7 \pm 0.2) \times 10^{-11} \mathrm{~cm}^{2}$, which is rather close to the average particle area of approximately $1.3 \times 10^{-11} \mathrm{~cm}^{2}$. These results not only confirm our conclusion that nanoparticles behaves like a $2 \mathrm{D}$ assembly of spherical nano-electrodes, but also provide a convenient electrochemical tool for accessing the number density of particles at the surface.

\section{Conclusions}

The electrostatic assembly of citrate stabilised Au nanocrystals of $18 \pm 2 \mathrm{~nm}$ diameter on pLys modified electrodes yields monodispersed randomly distributed 2D arrays. A maximum particle 
density of $(8.2 \pm 0.1) \times 10^{10} \mathrm{~cm}^{-2}$ separated by an average edge-to-edge distance of $25 \mathrm{~nm}$ is obtained after 4 hours of deposition in stagnant solution. The electrostatic interaction between the polyelectrolyte and the nanocrystals manifests itself by the development of a static dipole moment of the order of $2700 \mathrm{D}$ per particle. This dipole moment was estimated from the changes in the surface potential measured by ex situ Kelvin probe analysis. Considering the size of the nanocrystals, the apparent dipole moment corresponds to a displacement of $c a .5 \%$ of the average particle charge.

The absorption spectra of the nanoparticle assembly obtained in reflection mode exhibit a plasmon band red-shifted and considerable broader with respect to the spectrum of the particle in the aqueous suspension. The absorption spectra were analysed within the framework of the coherent-potential approximation, which allows evaluating the induced dipole moment in randomly occupied lattice of particles. This model was able to reproduce the spectral broadening to a some extent considering the interparticle distance estimated from the microscopic analysis. Furthermore, it was obtained that the red-shift of the plasmon band is determined by the interaction between the particles and the pLys layer.

Electrochemical studies in 1,2-dichloroethane show a strong electronic overlap between the nanocrystals and the metal substrate. The ferrocene oxidation at the formal redox potential appears controlled by the rate of electron tunnelling across the SAM and pLys layer in the absence of the Au nanoparticles. Upon electrostatic adsorption, the cyclic voltammogram recovered the diffusion-controlled features obtained for the bare metal substrate. Planar diffusion phenomena were observed due to the overlap of the spherical diffusion profiles generated around each particle. Finally, it is demonstrated that measurements of the double layer capacitance provide a simple mean for estimating the effective surface area of the nanocrystal assembly. The ensemble of the experimental results allows concluding that the particle assembly behaves as a $2 \mathrm{D}$ array of randomly distributed nano-electrodes.

\section{Acknowledgements}

We are indebted to Professor Hubert H. Girault, Director of the Laboratoire d'Electrochimie Physique et Analytique, for his encouragement and support. This work has been sponsored by the Fonds National Suisse de la Recherche Scientifique (Projects 2000-067050.01). We gratefully acknowledge Mr Brian Senior (CIME, EPFL) for the SEM images and Ms Valerie Devaud for the technical support. The Laboratoire d'Electrochimie Physique et Analytique is part of the European TMR network SUSANA (Supramolecular Self-Assembly of Interfacial Nanostructures).

\section{References}

1 A. N. Shipway, E. Katz and I. Willner, ChemPhysChem, 2000, 1, 18.

2 M. S. Gudiksen, L. J. Lauhon, J. Wang, D. C. Smith and C. M. Lieber, Nature, 2002, 415, 617.

3 G. Schmid, M. Baumle, I. Heim, M. Kroll, F. Muller and T. Sawitowski, J. Cluster Sci., 1999, $10,223$.

4 G. Schmid and G. L. Hornyak, Curr. Opin. Solid State Mater. Sci., 1997, 2, 204.

5 S. Carrara, in Nanoparticles and Nanostructured Films, ed. J. H. Fendler, Wiley-VCH, Weinheim, 1998, p. 349

6 E. Bakkers, A. L. Roest, A. W. Marsman, L. W. Jenneskens, L. I. de Jong-van Steensel, J. J. Kelly and D. Vanmaekelbergh, J. Phys. Chem. B, 2000, 104, 7266.

7 E. Bakkers, A. W. Marsman, L. W. Jenneskens and D. Vanmaekelbergh, Angew. Chem., Int. Ed. Engl, 2000, 39, 2297.

8 C. D. Bain, E. B. Troughton, Y.-T. Tao, J. Evall, G. M. Whitesides and R. G. Nuzzo, J. Am. Chem. Soc., 1989, 111, 321.

9 J. H. Fendler, Chem. Mater., 2001, 13, 3196.

10 C. J. Kiely, J. Fink, T. Hutchinson, J. G. Zheng, M. Brust, D. Bethell and D. J. Schiffrin, Electron Microscopy and Analysis 1999, 1999, 515.

11 S. Pethkar, M. Aslam, I. S. Mulla, P. Ganeshan and K. Vijayamohanan, J. Mater. Chem., 2001, 11, 1710.

12 M. Li, H. Schnablegger and S. Mann, Nature, 1999, 402, 393.

13 S. G. Hickey and D. J. Riley, J. Phys. Chem. B, 1999, 103, 4599.

14 S. G. Hickey, D. J. Riley and E. J. Tull, J. Phys. Chem. B, 2000, 104, 7623.

15 D. J. Riley and E. J. Tull, J. Electroanal. Chem., 2001, 504, 45.

16 E. Bakkers, J. J. Kelly and D. Vanmaekelbergh, J. Electroanal. Chem., 2000, 482, 48. 
18 Y. J. Liu, Y. X. Wang and R. O. Claus, Chem. Phys. Lett., 1998, 298, 315.

19 K. M. Chen, X. P. Jiang, L. C. Kimerling and P. T. Hammond, Langmuir, 2000, 16, 7825.

20 T. Cassagneau, J. H. Fendler and T. E. Mallouk, Langmuir, 2000, 16, 241.

21 K. Uosaki, T. Kondo, M. Okamura and W. Song, Faraday Discuss., 2002, 121, 373.

22 K. L. Kelly, E. Coronado, L. L. Zhao and G. C. Schatz, J. Phys. Chem. B, 2003, 107, 668.

23 P. Mulvaney, Langmuir, 1996, 12, 788.

24 C. Kittel, Introduction to Solid State Physics, Wiley, New York, 1986.

25 T. Baum, D. Bethell, M. Brust and D. J. Schiffrin, Langmuir, 1999, 866.

26 M. Lu, X. H. Li, B. Z. Yu and H. L. Li, J. Colloid Interface Sci., 2002, 248, 376.

27 J. F. Hicks, F. P. Zamborini and R. W. Murray, J. Phys. Chem. B, 2002, 106, 7751.

28 S. W. Chen and R. J. Pei, J. Am. Chem. Soc., 2001, 123, 10607.

29 S. W. Chen, R. W. Murray and S. W. Feldberg, J. Phys. Chem. B, 1998, 102, 9898.

30 S. W. Chen and R. W. Murray, J. Phys. Chem. B, 1999, 103, 9996.

31 S. W. Chen and Y. Y. Yang, J. Am. Chem. Soc., 2002, 124, 5280.

32 J. F. Hicks, D. T. Miles and R. W. Murray, J. Am. Chem. Soc., 2002, 124, 13322.

33 J. Turkevich, P. J. Stevenson and J. Hillier, Discuss. Faraday Soc., 1951, 11, 55.

34 J. J. Kakkassery, M. Carrara and D. J. Fermin, in preparation.

35 C. E. Jordan, B. L. Frey, S. Kornguth and R. M. Corn, Langmuir, 1994, 3642.

36 Y. Cheng and R. M. Corn, J. Phys. Chem. B, 1999, 103, 8726.

37 D. J. Fermín, H. Duong, Z. Ding, P.-F. Brevet and H. H. Girault, Phys. Chem. Chem. Phys., 1999, 1, 1461.

38 D. J. Fermín and R. Lahtinen, in Liquid interfaces in chemical, biological and pharmaceutical applications, ed. A. G. Volkov, Marcel Dekker Inc., Boca Raton, 2001, p. 179.

39 K. C. Grabar, P. C. Smith, M. D. Musick, J. A. Davis, D. G. Walter, M. A. Jackson, A. P. Guthrie and M. J. Natan, J. Am. Chem. Soc., 1996, 118, 1148.

40 J.-P. Abid, PhD Thesis, Swiss Federal Institute of Technology, in preparation.

41 M. Carrara, J.-P. Abid and D. J. Fermin, in preparation.

42 J. C. Maxwell Garnett, Philos. Trans. R. Soc. London, 1904, 203, 385.

43 J. C. Maxwell Garnett, Philos. Trans. R. Soc. London, 1906, 205, 237.

44 R. G. Barrera, G. Monsivais and W. L. Mochán, Phys. Rev. B, 1988, 38, 5371.

45 H. Shiba, Prog. Theor. Phys., 1971, 46, 77.

46 P. Soven, Phys. Rev. B, 1967, 156, 809.

47 A. Liebsch and B. N. J. Persson, J. Phys. C: Solid State Phys., 1983, 16, 5375.

48 P. B. Johnson and R. W. Christy, Phys. Rev. B, 1972, 6, 4370.

49 P. F. Luckham and J. J. Klein, J. Chem. Soc., Faraday Trans. 1, 1984, 80, 865.

50 B. R. Scharifker, J. Electroanal. Chem., 1988, 240, 61.

51 J. Meier, K. A. Friedrich and U. Stimming, Faraday Discuss., 2002, 121, 365.

52 J. Zhang, R. M. Lahtinen, K. Kontturi, P. R. Unwin and D. J. Schiffrin, Chem. Commun., $2001,1818$. 